Proceedings of the 2010 Winter Simulation Conference

B. Johansson, S. Jain, J. Montoya-Torres, J. Hugan, and E. Yücesan, eds.

\title{
REDUCING COMMUNICATION DETECTION AND EAVESDROPPING USING MOBILE AGENT RELAY NETWORKS
}

\author{
Hyon Kwak \\ Brett Borghetti \\ Air Force Institute of Technology \\ 2950 Hobson Way, WPAFB, OH 45433, USA
}

\begin{abstract}
Although mobile wireless communication provides connectivity where hardwired links are difficult or impractical, environmental conditions can still hinder communications. Increasing transmission power reduces battery life and increases susceptibility to eavesdropping. Adding stationary repeater nodes is impractical for highly mobile users in dangerous environments. Using remotely-controlled mobile relay nodes requires centralized control schemes which and adds network traffic overhead and introduces a single point of failure at the controller. An alternative is to create a Mobile Agent Relay Network (MARN). Each autonomous node in the MARN is an agent that decides where to move to maintain the network connectivity using only locally available information from onboard sensors and communication with in-range neighbor nodes. MARN agents form and maintain a communication network that provides connectivity for users while reducing the overall radio frequency footprint, minimizing the likelihood of detection and eavesdropping. We characterize the footprint reduction both theoretically and in simulation.
\end{abstract}

\section{INTRODUCTION}

Wireless communication has opened up many opportunities to relieve the challenges of wired communications, but it has introduced new challenges in the process. For users who need to remain connected for long periods of time or over long distances, power of their devices becomes an issue because battery life is limited. Interference due to natural or man-made causes can reduce the receiver's ability to receive signals from the transmitter - a fact which is exacerbated by mobile users as they pass through interfering terrain or near obstacles.

Military communicators add requirements and challenges to the design of any system. In an ideal situation, the military user would like to avoid eavesdropping (confidentiality) and would like to avoid revealing his position to unfriendly parties (stealthiness). Since wireless broadcasts are by definition not point-to-point, the potential for eavesdropping is a natural consequence. Even when using encoded transmissions, wireless broadcasters reveal their location to a wide audience each time they transmit.

There are several solutions which address a subset of these problems. Stationary wireless repeater stations with their own power source (much like cell-phone towers) could address the power challenge of wireless communication. These stations would still require reasonably powerful broadcasts and would thus not meet the stealthiness requirement, even if the transmissions were encoded. In addition, the stationary repeaters would not be able to move with the users as they executed their mission. Finally, stationary repeaters would present an attractive, easy target for opposing forces. 
Unmanned mobile vehicles could be used to mitigate some of the issues associated with static repeater stations: mobile platforms can move with the users as they execute their mission; mobile platforms are harder to find, fix, target, and kill, and thus make for harder targets. If enough of the unmanned vehicles (UV) were used, the transmission power for each could be reduced substantially, minimizing the probability of eavesdropping and increasing the stealthiness of each vehicle.

Unmanned vehicles bring new challenges to the domain. Unmanned vehicles must be controlled, and human operators are likely to be too scarce or expensive to be practical. If the UVs are centrally controlled, there will be overhead control traffic between the UV and the control station. The control station is a central point of failure (and thus a high value target for opposing forces) and the control traffic is subject to interference (deliberate or otherwise) just as is the regular communication traffic. While unmanned vehicles appear to be a better solution than static repeater stations, the concept of central control has several undesirable risks. For these reasons, this work explores UV solutions which are not centrally controlled.

When a non-centrally controlled solution is chosen, all sensing, deciding, and acting must be moved onto the UV platforms. The UV must sense its state, which includes the radio-frequency environment produced by nearby UVs plus any obstacles it needs to avoid. The UV must then decide what transmissions it will make and movements it will perform. Finally, the UV must execute those decisions with its communications gear and actuators. We designate a set of UVs that can perform all of these functions to provide communications connectivity for their users a Mobile Agent Relay Network (MARN).

\subsection{Characteristics of a desirable solution}

Before devising a solution for the mobile communication domain described above, it is important to formally outline the characteristics of a desirable solution. These requirements are divided into two domains: quality, and security. For clarity of discussion, the physical equipment of the solution is separated into user components and non-user components. User components are the items which the user carries or are collocated with the user (radios, cell phones, wireless devices). Non-user components comprise the connectivity backbone and include relay devices and associated mobile platforms for carrying the devices.

The goal of a communication solution is to keep the users connected to each other. A desirable communication solution for regular mobile users has the following quality characteristics:

- Connectivity: A valid solution is one in which all users are capable of communicating with each other. If this capability is temporarily unavailable because one or more users is out of communication, then the system is seeking a state in which full connectivity becomes available

- Overall Power Efficiency: Given two solutions A and B, solution A is preferred to solution B when solution A uses less overall power than solution B

- Generalized Robustness: Given two solutions, A and B, solution A is preferred to solution B if a non-user component can be removed from $\mathrm{A}$ without impacting the ability for the users to communicate while removing that component from B would impact connectivity with one or more users

For security, the following characteristics are also desired:

- Footprint/Eavesdropping Minimization: Minimize the physical area in which an unfriendly could detect a signal from the users or non-user components

- Communication robustness against malicious nodes: Minimize the impact that non-user equipment would have on the system's ability to remain connected if it was co-opted by adversaries (by a cyber attack, for example) 


\subsection{Contribution}

This paper focuses on quantifying the reduction of the signal footprint to be gained by using a MARN to form a relay network. By using a MARN (instead of increasing the power of the user's radios until they could communicate), the system maintains a reduced likelihood of detection and message interception. This finding is demonstrated analytically and through simulation.

The remainder of the paper is organized as follows. Section 2 reviews other solutions that could be used to provide connectivity for a group of users. In Section 3 we give a brief overview of how the MARN agents make decisions and discuss our approach for evaluating their signal footprint analytically and through simulation. Section 4 discusses the results of the simulation. Finally, in section 5 we conclude and provide recommendations for future work required to realize a MARN.

\section{RELATED WORK}

Section 1 provided several reasons why a centrally controlled mobile relay system was not suitable for a solving the communication task. In this section we discuss several alternatives to the central control model which could be used to solve the task. Each of these alternatives comes from the general class of distributed multi-agent systems attempting to solve a communication task.

Two types of tasks include the area coverage task and the mobile relay task. The area coverage task (often considered in wireless sensor network applications) solves the communication problem by providing maximal coverage of an area without concern for whether there are users within the area and where they are specifically located. The mobile relay task uses relays to provide connectivity between users (and possibly a base station) without concern for covering a specific physical area.

\subsection{Area Coverage Task}

In the sensor domain, the area coverage problem is defined as the maximization of the total area covered by sensors, often with the subgoal of minimizing redundancy (overlap) of coverage. There are many applications of area coverage, including tracking unfriendly targets, anomaly detection and location, and search and rescue. We present some of the decentralized approaches for area coverage below.

Coverage maps and history of visited locations: In this message-based coordination approach, each agent transmits its current coverage map and a history of the locations it has visited to its neighbors. Using this information, the agents take action attempting to strike a balance between agent dispersion (area coverage) and information gain (communication), while minimizing redundancy (repetitive coverage of an area) and time to convergence. Since each agent does not know in advance the planned movement of the neighbors, it infers their future movements based on history. The authors also consider noise in the environment and the potential of agent failure. Despite these challenges, the agents were able to provide area coverage (Cheng and Dasgupta 2007).

Light-weight beacons: In addition providing an approach for monitoring an area for intruders, this solution described in (Scheutz and Bauer 2006) addresses the problem of intercepting intruding enemies. Each friendly agent is equipped with two beacons necessary for interaction: one to repel friendly units and one to notify friendly units of intruders. This simple beacon-based system is more lightweight than previous works in this domain that relied on message-based communication. Each agent is able to sense the signal strength and directionality of their neighbors' beacons. With this configuration, the agents disperse to provide the necessary area coverage and intrusion detection. This work presents a reliable solution while being highly scalable and low in complexity and computing requirement.

Potential fields: In this work, the authors propose using virtual potential fields in multi-agent systems to address the area coverage problem (Howard, Mataric, and Sukhatme 2002). The potential field computation is based on the distance between two objects. If two objects are close, then the magnitude of the potential field repulsion would be greater than if two objects were far away. The 
potential field in this research causes intern-node separation as well as separation between the nodes and obstacles. Agents tend to spread themselves in the environment. Each agent is equipped with sensors such as laser or sonar and therefore capable of detecting each other and obstacles in the environment. Using the data from these sensors, each agent calculates the resulting potential field generated by its in-range neighbors and obstacles. Agents move according to their local calculation of the virtual potential field. This research presents a fast convergence and evenness of separation distance even with lack of explicit message-based coordination among the agents.

Deployment of static sensors by a mobile autonomous agent: In (Batalin and Sukhatme 2003), a mobile agent deploys static relay nodes (wireless sensor and relay devices) to solve the area coverage problem. Unlike the previous approach to area coverage which assumes there are a sufficient number of mobile units available, in this approach, there is only a single mobile agent operating in the area. This lone agent is capable of deploying a set of sensor/relay nodes into the explored environment and re-deploying new devices if a previously-deployed device stops working. The static nodes act as sensors in the environment and a guide for future exploration. If the environment changes or if a node is lost, the agent is recalled to investigate and (potentially) deploy new or replacement nodes. Assuming an unbounded supply of nodes (and time), this solution will provide both complete area coverage and network connectivity for any size environment.

\subsection{Mobile Relay Task}

The mobile relay task requires mobile agents to act as communication relays for the users of the network. Due to limitations in range and potential interference, as well as the unpredictable movements of the users in such network, users may experience temporary communication loss. A system of mobile communication relays could be used to maintain a wireless network to support the users. This type of solution is often used in Mobile Ad Hoc Networking (MANET) (Corson 1999). MANET protocols generally assume that nodes in the network act as both users and relays. Since users' movement (and thus the physical topology of the network) is not controllable, other solutions are required to reduce the likelihood of communication failures. We review several existing solutions.

Convoy formation: Maintaining a solid radio communication link between a mobile robot and its remote control station can be challenging as the robot enters a building. One approach to this presented in (Pezeshkian, Nguyen, and Burmeister 2006) is to deploy the robots in a convoy that includes one lead robot to be controlled by the control station and multiple autonomous relay robots. The rearmost relay robot will stop at the edge of the control station's signal range in order to maintain the connection back to the control station. Recursively, the rearmost relay robot still moving will then stop when it gets to the edge of the most recently stopped relay. The stopped relay robots can also be used to continue monitoring the area. If any stopped relay robot senses that it is no longer being utilized (due to network path shortcuts discovered by another deployed relay robot) then it will return to the convoy to be re-deployed at another location (Pezeshkian, Nguyen, and Burmeister 2006). The authors demonstrated, using real robots, the success of this approach in maintaining a connected network between the lead robot and the control station. Unfortunately, this approach assumes a single mobile robot using multiple relay robots to maintain a link to a single stationary control station. The method would build a single route and would not be able to build a network to service the more general mobile user scenario we are wishing to explore.

Local search algorithm for global optimal solution: Another approach explores the problem of using mobile relays that search for overall optimal network utility (Gerkey, Mailler, and Morisset 2006). The authors termed their relay agents as Commbots and use the global utility evaluation shown in Equation 1, with the following parameters:

- $C$ : set of $n$ Commbots

- $R(i, j)$ : an indicator function that returns 1 if there exists a route from $i$ to $j, 0$ otherwise 
- $Q(i, j)$ : real-valued function that returns the quality of the best route from $i$ to $j$. For the purpose of their simulation, they used the inverse of the square distance between $i$ and $j$ for $Q(i, j)$, as shown in Equation 2 .

$$
\begin{gathered}
U=\underset{i \in C j \in C, j \neq i}{ } R(i, j) Q(i, j)-\neg R(i, j) \\
\text { where } Q(i, j)=\frac{1}{\operatorname{dist}(i, j)^{2}}
\end{gathered}
$$

Using a local form of this utility function (without the outer summation), individual agents attempt to reach a local optimum utility. The authors used local annealing (modified simulated annealing), a modified distributed Breakout algorithm (modified Breakout algorithm); and an auction-based team formation. To constrain the search space of possible solutions, the authors discretized the environment and limited the movement range of each robot. The authors explain that the result of the experiments suggest additional information needs to be shared between the robots as well as possibility of exploiting patterns in the observed states in order to solve this problem more effectively (Gerkey, Mailler, and Morisset 2006).

\subsection{Summary of Related Work}

In this section, we reviewed several solution concepts for the area coverage task and the mobile relay task. Unfortunately none of these solutions meet the domain requirements of mobile users needing to maintain a low probability of detection network. The area coverage task assumes it is acceptable to flood the area with signal (thus using more power and increasing exposure to detection), while the mobile relay solutions assume either a fixed control station is available or there are no human users in the network which need to be supported. For these reasons we discuss a new approach in the next section.

\section{APPROACH}

A mobile agent relay network provides a radio-frequency-stealthy alternative to supporting the communications of mobile users. Each user carries a certain number of agents with them to the location where the communication network is desired. The users deploy the agents, and the agents would autonomously organize to form a network, even while the users continued to move. Once the initial network was formed, the agents would balance the need to maintain low-footprint connectivity with the need to increase robustness (or respond to failures) of other agents in the system.

The Mobile Agent Relay Network (MARN) has one goal: to establish and maintain communication connectivity for its users. This purpose can be broken down into two subgoals which are directly applicable to each agent. First, if an agent is not in connectivity with other agents, it needs to move within the physical space to locate other agents. Second, when in connectivity with other agents, an agent should position itself to obtain connectivity with users or other agents that can provide paths to the rest of the users.

One of the requirements for agents within a MARN is for the agents to stay within communication range of each other. This tendency must not bring the agents so close together that they don't provide adequate network coverage for their users. Algorithmic models for flocking behavior (Reynolds 1987) provide this delicately balanced behavior through two concepts known as cohesion and separation. In a flock of animals, cohesion is the tendency for the animals to stick together to reap the benefits of the security of the group. Separation is the tendency for agents (or their biological inspiration) to avoid colliding with each other - an act which wastes energy. When moving as a flock, these two desires, along with alignment (the desire to head in the general direction that the flock is heading) are 
used to produce a desired velocity vector for each entity using only inputs from nearby neighbors. When all of the agents within a simulated flock obey these rules, the result is a realistic simulation of the behaviors of flocking animals. For this work, separation and cohesion are used to drive the agent's behavior based on observations of their neighbors as outlined in (Kwak 2010).

In the approach used for this research, the agents are subject to several simplifying assumptions that would not necessarily be true in a corresponding physical domain:

- Each agent knows the relative vector (distance and heading) to each other user or agent that is within communication range (its neighbors). Some combination of localization and communication is assumed to be available to the agents.

- Computational ability and memory for each agent is unbounded. Real time response is not a requirement (although the calculations and memory used in (Kwak 2010) are $O\left(n^{2}\right)$ and are computable in real time for the numbers of agents and users likely to be seen in use.)

\subsection{Analytical Evaluation}

A simple problem in the theoretical domain is to create a smallest-signal footprint network of agents that provides connectivity for two non-co-located users. In theory, given an infinite number of agents in a line between the two users, the transmission power could be reduced to an arbitrarily small value, allowing an arbitrarily small footprint for each agent. The signal footprint of the overall network could be made arbitrarily close to that of a wired network with one wire between the two users.

While this result is the best possible result from a signal footprint consideration, it is not practical for the users to carry an unbounded number of agents. For this work, we instead focus on a reasonable number of agents for each user to carry while still providing enough agents that connectivity is possible and the signal footprint is kept small. We now analyze optimal solutions to the simple problem described previously with a small number of agents per user.

The total area of footprint for two equal radius $r$ overlapping circles is given by

$$
2 r^{2}-\left(\frac{2 r^{2}}{3}-\frac{r^{2} \sqrt{3}}{2}\right)
$$

where the first term represents the sum of the area of the circles, and the second term represents the overlap of the two circles. We can compute the total footprint for an arbitrary number of overlapping circles on a line between two users by dividing the line between the two users into equal segments and placing agents between each segment. The communication radius for each agent and user is the segment length. Figure 1 shows the coverage ratios of various numbers of agents with respect to the scenario with no agents.

Although it is theoretically possible to achieve an order of magnitude reduction of the signal footprint using as few as 3 agents per user, this is an analysis of the optimal end state of a static system. The agents need to search for other agents and users when they are first deployed, and even when they converge on a network solution it is unlikely to be optimal. The next portion of the paper discusses the simulation used and the experiments designed to explore the footprint generated by dynamic MARN agents.

\subsection{Simulation}

For testing the performance of the agents, the simulation used in (Kwak 2010) was extended to capture the area of the overall signal footprint generated by the agents and users. The simulator runs on the Processing Development Environment (Fry and Reas 2010) and was coded in the Eclipse IDE with JavaSE-1.6 library.

Several assumptions were made during the development of the simulator:

- The simulation is two-dimensional (only X and Y dimensions are modelled). 


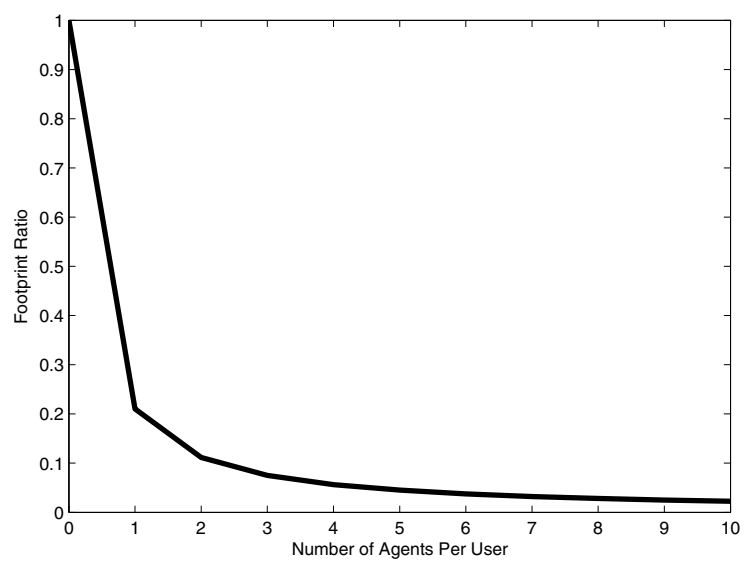

Figure 1: Analytical results for radio coverage ratio for MARN agents. The ratio indicates the likelihood a MARNbased system with that number of agents per user would have been subject to eavesdropping (compared to the original communication system without any MARN agents).

- Signal propagation is equal in all directions (circular).

- Signal reception is binary (either the agent is in range or it is not): signal strength, noise, and errors are not modelled.

- Transmission range is equivalent to receive range.

- Simulation entities (users and agents) are modelled as point-masses. The X and Y position of each entity are tracked using Java's 'float' precision variable, as are their velocities and acceleration.

- Forces are applied to entities to determine entity acceleration, and the environment is frictionless. There are limits on the maximum force that can be exerted on- and the maximum velocity of the agents.

- Simulation updates occur at discrete time intervals: each agent is given an update, queried for its decision, then all decisions are acted upon simultaneously (by the simulation controller) before the next time step.

\subsection{Experiment}

The goal of this research is to show that a autonomous relay agents can be used to reduce the signal footprint required if they were not present. While the previous discussion showed a limit on the minimum footprint obtainable with a given number of agents, the analysis is not complete without a characterization of the footprint during the operation of the MARN. This portion of the paper addresses the experimental results during MARN operation.

The simulator is used to experimentally measure the signal footprint of the agents and users. In each run, there are two users and several agents per user. The footprint ratio measurement is given by

$$
\frac{U_{1} \cup U_{2} \cup A_{1} \cup A_{2} \cup \cdots \cup A_{n}}{U_{1} \cup U_{2}}
$$

where $U_{i}$ and $A_{j}$ are the footprint circles for the specified two users and $n$ agent entities respectively.

Two parameters are varied in these experiments: the number of agents each user starts with, and the fixed transmission radius of each agent. The values for each of these parameters was chosen after a pilot study which revealed what settings enabled the MARN agents to form a network at least $80 \%$ of the time. The number of agents per user was varied from four to ten ( 7 settings). The fixed transmission radius of each agent was varied from $25 \%$ to $50 \%$ of the original user's transmission radius ( 4 settings). 
An experimental scenario consists of an agent-per-user-count and a transmission-radius. A total of 28 scenarios were evaluated.

Each scenario was repeated for 30 trials. Each trial ended when either the agents failed to provide a network for the users within 2000 timesteps (unsuccessful) or the agents succeeded in forming a network before the time limit (successful). For successful trials the footprint ratios were approximated by sampling the space. The results of the successful trials are reported in the next section.

\section{RESULTS}

We first explored the dynamics of the MARN system in terms of the change of the user and agent footprint ratio. Figure 2 shows the change in average footprint ratio for four agents as they form a network, and Figure 3 shows the same for eight agents. At the start of each scenario before the agents are deployed, the total footprint is a small fraction of the footprint that would be required if the agents were not available. Shortly after the agents are deployed they spread out from the user, increasing the footprint. Then, as the agents begin to encounter agents deployed by the other user, they begin to form a partial network and the footprint decreases. Finally, when most of the agents are part of the network, the footprint is at its operational minimum.

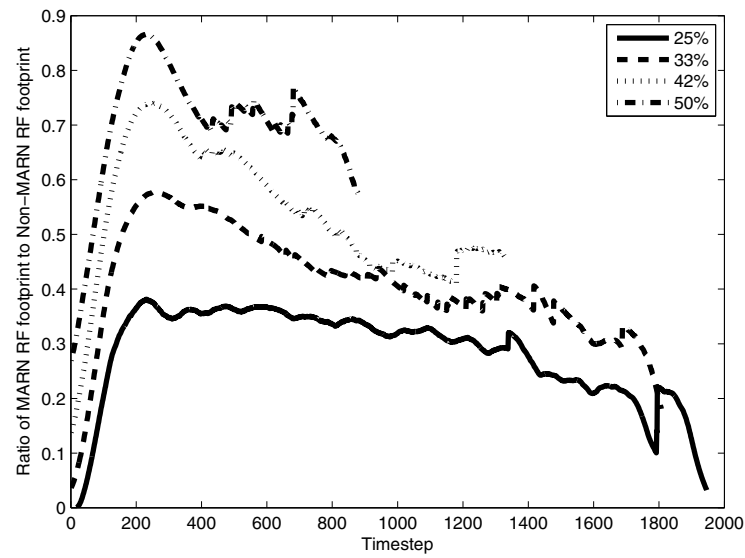

Figure 2: Mean radio coverage ratio for 4 MARN agents per user at four power settings (averaged over 30 trials). The percentages for each line indicate the percentage of the original user's radius used for each MARN agent. The ratio indicates the likelihood the MARN-based system would have been subject to eavesdropping (compared to the original communication system without MARN).

As expected, when four agents are used (Figure 2), the time for the agents to build the network is somewhat longer than when eight are used (Figure 3). The other scenarios with five, six, seven, nine, and ten agents (not shown) followed this trend: the more agents in the system, the faster the network was created.

Another interesting, but expected phenomenon confirmed by the results shown in Figure 2 is that the larger the communication radius, the quicker the agents are able to form a network. Unfortunately, this expediency of network formation is not without a cost. The more powerful transmissions cause a larger footprint, making the network more susceptible to detection and eavesdropping.

\section{CONCLUSIONS AND FUTURE WORK}

In this work, our goal was to show that a Mobile Agent Relay Network (MARN) could be used to reduce the signal footprint (and thus probability of detection) of a set of users by allowing them to reduce their transmission strength and instead rely on autonomous relays to self organize a network. Through theoretical analysis of the domain, we developed a minimum required footprint ratio. Simulations of 28 scenarios provided evidence that even during the search phase of self-organizing, the agents 


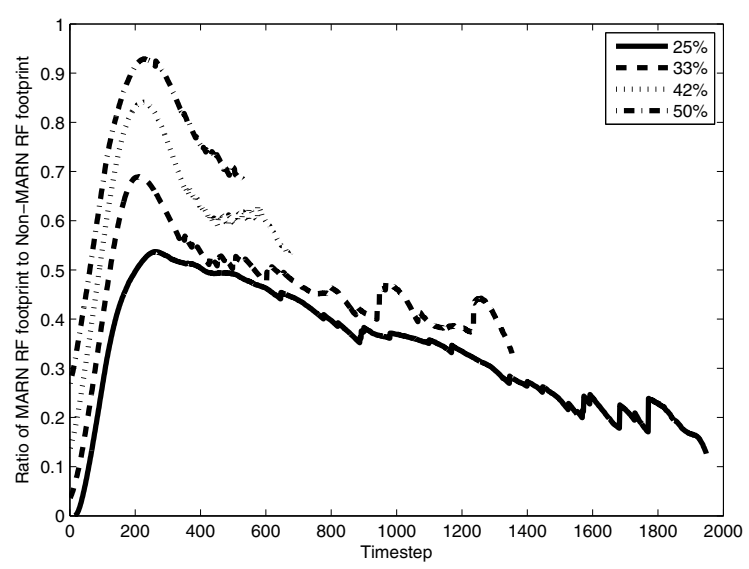

Figure 3: Mean radio coverage ratio for 8 MARN agents per user at four power settings (averaged over 30 trials). The percentages for each line indicate the percentage of the original user's radius used for each MARN agent. The ratio indicates the likelihood the MARN-based system would have been subject to eavesdropping (compared to the original communication system without MARN).

maintained a footprint that was a fraction of what the original users would have required without MARN.

There are several areas for future exploration. In this work, only two users were present, and they did not move during the scenario. There were also no intervening obstacles or other radio-frequency phenomenon present in the system. In the future, it will be important to characterize the behavior of the MARN with more users that are mobile in a more realistic environment. This work also used only analytical techniques and simulated experiments to provide support. Future efforts would need to explore implementation on robotic relays in various environments. Finally, this research assumed that all of the agents in the system were fully functional and trustworthy. While (Kwak 2010) began exploring the effect of malicious nodes in his thesis, there is more work to be done on determining how robust the MARN is to such attacks.

\section{REFERENCES}

Batalin, M. A., and G. S. Sukhatme. 2003. Coverage, exploration and deployment by a mobile robot and communication network. In Telecommunication Systems Journal, Special Issue on Wireless Sensor Networks, 376-391.

Cheng, K., and P. Dasgupta. 2007. Dynamic area coverage using faulty multi-agent swarms. In IAT '07: Proceedings of the 2007 IEEE/WIC/ACM International Conference on Intelligent Agent Technology, 17-23. Washington, DC, USA: IEEE Computer Society.

Corson, S. 1999. Mobile Ad hoc Networking (MANET): Routing Protocol Performance Issues and Evaluation Considerations. RFC 2501 (Informational).

Fry, B., and C. Reas. 2010, February. Processing. http: / / processing . org.

Gerkey, B. P., R. Mailler, and B. Morisset. 2006, Jul. Commbots: Distributed control of mobile communication relays. In Proc. of the AAAI Workshop on Auction Mechanisms for Robot Coordination (AuctionBots), $51-57$.

Howard, A., M. J. Mataric, and G. S. Sukhatme. 2002. Mobile sensor network deployment using potential fields: A distributed, scalable solution to the area coverage problem. In 6th International Symposium on Distributed Autonomous Robotics Systems (DARS02), 299-308.

Kwak, H. 2010. Toward a mobile agent relay network. Master's thesis, Air Force Institute of Technology.

Pezeshkian, N., H. G. Nguyen, and A. Burmeister. 2006. Unmanned ground vehicle non-line-of-sight operations using relaying radios. In IASTED Robotics and Applications (RA 2006), 17-20. 
Reynolds, C. W. 1987. Flocks, herds and schools: A distributed behavioral model. In SIGGRAPH '87: Proceedings of the 14th annual conference on Computer graphics and interactive techniques, 25-34.

Scheutz, M., and M. Bauer. 2006, Oct. A scalable, robust, ultra-low complexity agent swarm for area coverage and interception tasks. In Computer Aided Control System Design, 2006 IEEE International Conference on Control Applications, 2006 IEEE International Symposium on Intelligent Control, 2006 IEEE, 1258 -1263.

\section{AUTHOR BIOGRAPHIES}

HYON KWAK earned a M.S. degree in Computer Science in 2010 from the Air Force Institute of Technology in Dayton, OH; and a B.S. in Computer Science in 2002 from Illinois State University, Normal, IL. Capt Hyon Kwak is serving as an officer in the US Air Force. His research interests include artificial intelligence, multi-agent systems and machine learning. His email address is<hyon.kwak@gmail.com>.

BRETT BORGHETTI earned a Ph.D. in Computer Science in 2008 from the University of Minnesota, Twin Cities, MN; a M.S. degree in Computer Systems in 1996 from the Air Force Institute of Technology in Dayton, OH; and a B.S. in Electrical Engineering in 1992 from Worcester Polytechnic Institute (WPI), Worcester, MA. Lt Col Borghetti is an Assistant Professor of Computer Science at AFIT. His research interests focus on artificial intelligence, multi-agent systems, game theory, and machine learning. His email address is <brett.borghetti@afit.edu>. 